\title{
Dissecting cadaveric middle ear: approaches
}

\begin{abstract}
Introduction: The temporal bone is a valuable resource for the study of diseases of middle ear and teaching anatomy to both undergraduate as well as post graduates. It is one of the most complicated bones of skull and lodges in it the special sense organ for hearing and balancing and associated nerves.
\end{abstract}

Objectives: Various books and dissection manuals described different approaches to middle ear along with intrapetrous course of facial nerve. We have discussed different approaches to middle ear, their benefits and drawbacks

Data synthesis: Different approaches to middle ear cavity have been described by different dissection manuals and various authors. Every method or approaches have their own merits and demerits.

Conclusion: Dissection on decalcified temporal bone and tegmen tympani approach seems to be the best tool for studying middle ear, course of facial nerve inside the temporal bone as well as preparing specimen.

Keywords: temporal bone, dissection middle ear, tegmen tympani
Volume 2 Issue 5 - 2016

Krishna Pandey, Nishtha Singh, Badal Singh

Department of Anatomy, MLN Medical College, India

Correspondence: Krishna Pandey, Department of Anatomy, MLN Medical College, Allahabad, India, Email kp260683@gmail.com

Received: May 14, 2016 | Published: May 26, 2016

\section{Introduction}

Surgical training as well as teaching methods has shown a technologically driven evolution. ${ }^{1}$ Simulation and Synthetic replicas of temporal bones made of acrylic resins are used for trainee ENT Surgeons. ${ }^{2,3}$ But the traditional dissection on temporal bones remains simple, feasible, and convenient teaching tool despite technical advancements. We as an Anatomist promote and encourage the training of our future surgeons by the use of cadaveric temporal bone dissection via different approaches. Learning by hands on training is considered ideal. ${ }^{1}$ The temporal bone has a very complex configuration, containing vital structures known for their anatomical variations. Different types of pneumatisation of mastoid air cells, Korner's septum, facial nerve and ossicular anomalies, low lying dura and anteriorly placed sigmoid sinus and various other anomalies yet unnoticed. Dissection on cadaveric bones will suffice to study of anomalies as well as normal anatomy of this region. Cawthorne has stated that facial nerve surgery needs experience and should be performed only after practice on human cadaveric temporal bones. ${ }^{4}$

\section{Different approaches to middle ear}

Middle ear cavity is a mucous lined air filled irregular space within the petrous part of temporal bone compared to a cube, containing ear ossicles and associated muscles. We can reach it via lateral approach or via dorsal and dorsomedial approaches. ${ }^{5}$

Kamal Singh et al. ${ }^{6}$ described an innovative procedure-Kobbler's cut .By this method one can quickly expose the middle ear cavity and procure the ear ossicles (Malleus, Incus \& Stapes).Moreover, the view of the internal structures and anatomical features of the middle ear cavity are more apparent. The ossicles so received can be used for potential further study for forensic aspects ${ }^{7}$ as well as ENT prospective. This method proves to be the easiest, less time consuming and least destructive method of dissection as compared to the traditional methods of piece meal removal of bone, chipping away the roof (tegmen tympani) or snipping off done for exploring the middle ear cavity and mastoid air cells. ${ }^{8}$
Lisowski $\mathrm{FP}^{9}$ describes that for the study of the auditory and vestibular apparatus, the temporal bone and the adjacent parts of the sphenoid and occipital bones has to be removed and decalcified in $10 \%$ concentrated solution of nitric acid, after following the full procedure of decalcification bone becomes soft and easy to dissect. After examining the prosected part bone is gently removed between the hiatus for the greater petrosal nerve and the geniculate ganglion; this exposes the course of the nerve and also the coils of the cochlea, followed by removal of tegmen tympani exposing the small auditory ossicles. This procedure seems best for specimen preparation as well as for detailed study of both internal as well as middle ear, but it is more time taking and will require a patient trainee.

Cunninghams's manual ${ }^{10}$ described tegmen tympani approach, with a point of rigid knife or seeker a small aperture is made then it is lifted up with seeker and breaking of tegmen tympani is done first in anteromedial direction then in the posterolateral direction towards the junction of transverse and sigmoid sinus. This approach is conventional and little less precise as the bone may not fracture in a desired direction cut edges may create confusion with ear ossicle as they are also situated within the bone.

Repetitive laboratory dissection of human temporal bone specimens creates an eye-hand familiarity that is essential for safe operating in the live patient. For post graduate training on temporal bone surgeries there should be well equipped laboratories in various colleges that use, high-speed otologic drill (pneumatic or electric), along with Irrigating suctions, Hosing for both suction and irrigation, No. 15 blade scalpel, Heavy periosteal elevator, Round knife, Annulus elevator, Curved needle, microscissor's all these instruments suffice in practicing for surgeries related to ear specially for those who opt for specialisation in ENT.

Grant's dissector describes approach to middle ear via tegmen tympani. ${ }^{11}$

Now a days, the surgical procedures of the middle ear are traditionally carried out with the help of microscopes, with few limitations (Less visualisation of deep and lateral recess). ${ }^{12}$ 
The surgical use of endoscopes in otology is still very restrict and their application in most cases is merely secondary to the use of microscopes, but for specialist training it is must. The middle ear endoscopic dissection by transcanal and modified transcanal ways is possible and enables an excellent view of important structures in the middle ear. The ear surgery techniques with endoscope are currently similar to the microscopic ones, but some instrument adaptation, new techniques and familiarization with such appliances are critical for the future procedures and the popularization of the ear endoscopic surgery. ${ }^{13}$

\section{Conclusion}

Study of middle ear cavity is possible via different approaches as well as via endoscope and simulation devices. Every method has its own pros and cons as discussed above. As an Anatomist we prefer dissection on decalcified temporal bone and roof approach to middle ear, which is very appropriate for specimen preparation also, the only drawback is that it is more time consuming. For surgical practice of Otorhynolaryngologist bone dissection should be sufficed with endoscopic training, temporal bone dissection laboratories and training on simulation devices.

\section{Acknowledgements}

None.

\section{Conflict of interest}

Author declares that there is no conflict of interest.

\section{References}

1. Naik MS, Naik SM, Bains NK. Cadaveric Temporal Bone Dissection: Is It obsolete Today? Int Arch Otorhinolaryngol. 2014;18(1):63-67.
2. Wiet GJ, Rastatter JC, Bapna S, et al. Training otologic surgical skills through simulation-moving toward validation: a pilot study and lessons learned. J Grad Med Educ. 2009;1(1):61-66.

3. Okada DM, de Sousa AM, Huertas Rde A, et al. Surgical simulator for temporal bone dissection training. Braz $J$ Otorhinolaryngol. 2010;76(5):575-578.

4. Cawthorne T. The surgery of the temporal bone. Ann R Coll Surg Engl. 1953;12(6):403-423.

5. Singh K, Sirohiwal BL, Rohilla A, et al. Cobbler's Cut: An Innovative Technique for Middle Ear Dissection. Journal of Forensic Res. 2014;5:222.

6. Cattell HW. Postmortem Pathology: A Manual of the Technic of PostMortem Examination and the Interpretations. 3rd ed. Philadelphia: JB Lippincott Company; 1906. p. 272-274.

7. Warthin AS. Practical Pathology: A Manual of Autopsy and Laboratory Technique. 2nd ed. Ann Arbor: George Wahr Publisher; 1911:81-85.

8. Singh K, Chhabra S, Lal Sirohiwal B, et al. Morphometry of Malleus a Possible Tool in Sex Determination. J Forensic Res. 2012;3:6.

9. Lisowski LP. A Guide to Dissection of the Human body. 2nd ed. Singapore: Singapore University Press; 2004. p. 395-400.

10. Romanes GJ. Cunningham's Manual of Practical Anatomy. Oxford, New York; 2015. p. 173-174.

11. Patrick Tank W. Grant's Dissector (13). USA: Lippincott Williams and Wilkin's; 2005. p. 208-210.

12. Tarabichi M. Endoscopic management of Cholesteatoma: long term results. Otolaryngol Head Neck Surg. 2000;122:874-881.

13. Nogueira Júnior JF, Cruz DN. Ear Endoscopic Surgery: Dissection of the Middle Ear Intl. Arch Otorhinolaryngol. 2009;13(4):421-425. 